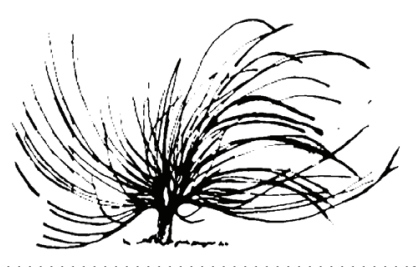

\title{
La competencia léxico-productiva como predictor del desempeño académico
}

\author{
Martín Gonzalo Zapico ${ }^{1}$ \\ Universidad Nacional de Mar del Plata \\ Instituto Nacional de Formación Docente Continua \\ Mar del Plata, Buenos Aires \\ athenspierre@gmail.com \\ Marcos Gabriel Zapico ${ }^{2}$ \\ Universidad Nacional de Mar del Plata \\ Mar del Plata, Buenos Aires \\ copotopo@hotmail.com
}

\begin{abstract}
Resumen
El desempeño académico en el nivel superior, específicamente la posibilidad de predecirlo, es un tema de enorme profusión en términos tanto de teoría como de práctica. Hay un acuerdo entre quienes investigan de que, dentro de lo difícil que es establecer factores predictivos, tres tienen un grado moderado, pero no concluyente de correlación: el desempeño previo en el
\end{abstract}

\section{(c) (1) $\Theta \Theta$}

Recibido: 6 de julio de 2017—Aprobado: 21 de mayo de 2018

http://dx.doi.org/10.15359/rep.13-1.10

1 Profesor en Letras de la Universidad Nacional de Mar del Plata. Actualmente se desempeña como docente-investigador en las cátedras de Introducción a los Estudios Literarios y Teoría Literaria I. Ha publicado variedades de artículos en las áreas de lingüística, educación y psicología, y asistido a numerosos congresos de dichas disciplinas.

2 Alumno avanzado de la carrera de Licenciatura en Bioquímica de la Universidad Nacional de Mar del Plata, donde se desempeña como ayudante de cátedra en el área de Química. Ha publicado en el área de educación, específicamente en la relación ciencias exactas-didáctica, además de asistido a diversas actividades y congresos. 
nivel secundario, la motivación en todos sus matices y el juicio crítico. La hipótesis de este trabajo fue que el conocimiento léxico productivo de un sujeto, que puede ser evaluado en términos de sinonimia, es un factor que determinará el rendimiento académico. Para dicho propósito se realizó una comparación cuantitativa (estadística descriptiva y correlacional) en el desempeño obtenido por cuatro grupos de estudiantes de los profesorados de Letras, Inglés, Educación Primaria e Historia en sus respectivas materias introductorias del Instituto de Formación Docente Continua (IFDC, situado en San Luis, Argentina), a los cuales previamente se les había administrado un test de sinónimos. Los resultados sugieren que el conocimiento léxico productivo, evaluado en términos de sinonimia, es un factor que permite predecir el rendimiento académico $y$, por ende, es pertinente considerar la implementación de programas orientados a la promoción de esta competencia en los cursos introductorios de las carreras de nivel superior.

Palabras clave: Evaluación, desempeño académico, vocabulario productivo, educación superior.

\begin{abstract}
Academic performance at the high level, specifically the ability to predict it, is an issue of enormous profusion in terms of both theory and practice. There is an agreement among researchers that in how difficult it is as part of the difficulty to establish predictors, three of them have a moderate but inconclusive correlation degree: past performance in high-school level, motivation in all of its nuances, and critical judgment. The hypothesis of this paper is that the productive lexical knowledge of a subject, which can be evaluated in terms of synonymy, is a factor that will determine the academic performance. For this purpose quantitative comparison (descriptive and correlational statistics) of the performance obtained by four groups of students of professorships Language, English, Primary education and History in their respective introductory courses of the Instituto de Formación Docente Continua (IFDC located in San Luis, Argentina), whom previously had been administered a
\end{abstract}


test of synonyms, was performed. The results suggest that productive lexical knowledge, assessed in terms of synonymy, is a factor that predicts academic performance, and therefore it is pertinent to consider implementing it aimed at promoting this competence in the introductory courses of top level education programs.

Keywords: evaluation, academic performance, productive lexical, higher education

\section{Presentación y justificación del tema}

El abordaje de la temática seleccionada se relaciona íntimamente con un problema que atañe a la comunidad educativa en general y al nivel superior en particular: la alta tasa de deserción en primer año. Este fenómeno ha sido reportado numerosas veces, tanto en comunicaciones formales como artículos y libros (Barsky, Sigal y Dávila, 2012; Giovagnoli, 2001) como en seminarios, ponencias e incluso charlas cotidianas con gran cantidad de docentes de distintas instituciones, tanto universidades nacionales como institutos de formación docente.

Esta alta tasa de deserción, que se atribuye a falencias en la formación previa (realidad que ha sido documentada en estudios como García de Fanelli, 2014) exige de las investigaciones en educación soluciones, tanto a corto como largo plazo, para sanear el estado de la situación. A la luz de que la alta tasa de deserción es solo parcialmente atribuida a una formación secundaria defectuosa y de que se han estudiado la incidencia de otros factores tales como el nivel socio-económico, la estructura de contención afectiva, las expectativas, entre otros; queda abierta la posibilidad de que los mismos establecimientos de nivel superior operen con acciones concretas a fin de mejorar el rendimiento de sus estudiantes, así como las posibilidades de permanencia en la carrera.

Por otro lado, se ha puesto en relieve que los factores que más parecen predecir el desempeño académico en las carreras de grado son fundamentalmente tres: el desempeño previo, la motivación en muchos de sus matices y la capacidad de juicio crítico. No obstante, estos tres presentan un inconveniente severo a la hora de ser empleados para diseñar e implementar políticas educativas de mejora: son tres factores sobre los que no hay tiempo para actuar en primer año, puesto que se 
relacionan íntimamente con la formación previa y la experiencia de vida del sujeto, ambos escenarios que escapan a las instituciones.

A raíz de esta imposibilidad y tomando como base investigaciones previas, se postula la presencia de otro posible predictor: la competencia léxico-productiva. Esta, que puede ser descrita brevemente como la capacidad de producir textos escritos/orales en una lengua determinada, tiene una ventaja respecto a las tres mencionadas anteriormente: puede ser analizada, medida y mejorada a través de la implementación de programas de alfabetización y cursos intensivos en el período de ingreso a la universidad sin la necesidad de invertir en profesionales de la salud (como es el caso de la motivación que requeriría la presencia y trabajo prolongado de psicología) o del servicio social (que requeriría la presencia y trabajo prolongado de asistentes sociales). Claro está, no es que los otros factores sean prescindibles (todo lo contrario ya que han demostrado ser efectivos como variables predictivas), es que para su tratamiento se requiere mucho tiempo y en el trasncurso de ese tiempo es que sucede la deserción.

A partir de esta investigación se busca perfilar cada vez más a la competencia léxico-productiva (la cual medimos en términos de sinonimia) como un predictor del desempeño académico. Si así resultare ser, esto daría la pauta para la implementación de políticas educativas concretas dictadas por especialistas que permitirían tratar (al menos en una de sus aristas) la problemática de la deserción. Puesto que si el estudiantado que tiene un buen desempeño es el que mejor puntúa en los test de sinonimia, solo hay que lograr que más estudiantes tengan las capacidades que dichos test evalúan para lograr una mejora.

\section{Estado del arte}

La evaluación del desempeño académico comprende una gran cantidad de métodos y acciones cuya finalidad consiste en reflexionar de manera sistemática sobre los resultados que obtiene el alumnado a lo largo de su trayecto por el sistema educativo en sus distintos niveles (primario, secundario, superior) así como en sus distintas modalidades (educación informal, no formal y formal) aunque se centra fundamentalmente en esta última (Cozza, 1998).

Se ha trabajado sobre el concepto en numerosos artículos e investigaciones. Tradicionalmente se ha pensado la evaluación como la 
implementación de pruebas, la recogida de información y la conformación de un juicio valorativo de la misma a raíz de una variedad de criterios, según los propósitos de formación (González-Pérez, 2000). A este respecto se hace saber que el proceso de evaluación no es un evento aislado en el cual interviene solo el sujeto evaluado, sino que implica a toda la comunidad educativa: la institución, su personal directivo y docente y sus estudiantes.

Además, dicho proceso no es un acontecimiento neutro o desproblematizado. Se han cuestionado, por ejemplo, las distintas formas de evaluar (Arribas, 2012) en función de que la evaluación tiene dos grandes propósitos: la función formativa en la cual nos hallamos ante una instancia más de aprendizaje donde tanto docentes como estudiantes tienen algo que aprender; y la función certificadora cuyo propósitos es dar cuenta de que un sujeto está en conocimiento de un saber particular y definido. Es decir, la forma de evaluar está ineludiblemente condicionada por lo planteado anteriormente. Ante esta situación se ha propuesto que la evaluación más que un suceso aislado debe tener la misma condición que el proceso de enseñanza-aprendizaje, un continuum que comprende no solo el desempeño en una instancia única sino que abarca desde las prácticas áulicas diarias, los trabajos realizados a lo largo de la cursada hasta la evaluación particular que no debe ser dejada de lado. La conclusión de que la evaluación continua (en oposición a la final y la mixta) da mejores resultados en términos de rendimiento del alumnado ha sido también reportada en otras investigaciones (Buscá et al., 2011; López, 2008).

También se han problematizado los efectos de realizar una evaluación inadecuada e inconsistente, donde no hay coherencia entre aquello que se enseña y lo que se pretende evaluar. Esta fuerte crítica a la denominada racionalidad ténica viene a ser reemplazada por un nuevo paradigma en evaluación: la racionalidad práctica, que ya es una realidad en la mayoría de los programas y planificaciones a nivel nacional. Sus características fundamentales son la horizontalidad en la circulación de qué se va a evaluar y cómo, la gran implicación del profesorado que se compromete en el proceso de evaluación en vez de alejarse y limitarse a ser juez o jueza imparcial, el dinamismo, y la prioridad que tiene el proceso de aprendizaje como tal, en vez de la puntualidad del examen (Álvar-Méndez, 2001). La falta de compromiso ante estas premisas (sea por parte de la institución o del cuerpo docente) 
conlleva al fracaso de la evaluación y, en general, al fracaso escolar del alumnado, promoviendo quizá, sin querer, la exclusión académica en sus distintos niveles.

En el marco de la evaluación, entonces, es apropiado hablar del rendimiento académico o desempeño académico. Se está ante un tema absolutamente complejo tanto en términos de problemáticas que comprende (la deserción en los distintos niveles escolares (Rodríguez, Pita y Torrado, 2004; Tejedor y Repiso, 2007; Vélez, Schiefelbein y Valenzuela, 1994), la tasa de finalización de carrera, los problemas en torno a la motivación (Pozo, Álvarez, Contreras y Reséndiz, 2008), las relaciones entre desempeño y contexto (Meerbeke y González, 2005); definiciones sobre lo que es evaluar y qué es el desempeño; las distintas formas de evaluación (Arribas, 2012); las críticas a la evaluación (Pérez, 2001) precisar que es un buen desempeño o uno malo (Méndez 2001; Navarro, 2003), como metodologías de investigación (estudios cuantitativos longitudinales [Jiménez, Izquierdo y Blanco, 2000], estudios de cohorte [Renault, Solano y Kohan, 2001], seguimiento, análisis de entrevistas y percepciones [Vargas, 2007]. etc) y hasta campos de aplicación (investigar para desarrollar políticas educativas, para sanear una situación concreta, para obtener un panorama de la cuestión, etc).

Por eso, ante todo es pertinente acotar qué es exactamente aquello que estamos investigando: la posibilidad de predecir el desempeño en términos de éxito académico a partir de conocer la competencia léxica productiva de un sujeto. Diversos estudios (Benitez, Giménez y Osicka, 2000; Garaigordobil y Torres, 1996; Lugo, Villatoro, Medina-Mora y García, 1996; Pérez, 2002; Pozo y Álvarez, 2008) muestran que son fundamentalmente tres los parámetros que tienen una alta tasa de fiabilidad para predecir el desmpeño académico: el desempeño previo (alumnado que en el nivel secundario ha tenido una alta tasa de éxito y ha concluido con un buen promedio suele sostener el rendimiento y adaptarse sin problemas al primer año de una carrera), la motivación (alumnado que estudia carreras que ha elegido como primera opción, con altas expectativas sobre el futuro y optimismo, con baja ansiedad, suele tener alta tasa de éxito en el primer año) y el juicio crítico (alumnado que puntua alto en los test de pensamiento crítico, es decir, que posee una capacidad de comprensión lógica alta, suele tener un buen desempeño). 
Aún así, en esas mismas investigaciones se aclara que otras muestran resultados que parecen contradecir lo planteado en el párrafo anterior. Esto pone de manifiesto que los tres parámetros señalados no son suficientemente fiables como para hablar de una correlación alta entre ellos y el desmempeño académico.

La idea de que el conocimiento léxico podría llegar a perfilarse como un predictor fiable del desempeño académico surge a partir de investigaciones previas (especialmente Zapico, 2016a) donde se mostró la importancia de las competencias generales para llevar adelante un buen rendimiento. El vocabulario como gran constructo puede ser analizado en dos planos diferentes: el de la recepción y el de la producción. El de la recepción tiene que ver con las competencias que tiene un sujeto para interpretar un texto codificado mediante un sistema simbólico cualquiera (en lo que compete a educación, el idioma en el cual se comunican docente-alumnado así como de los textos) a partir del reconocimiento de los relaciones sintagmáticas y paradigmáticas fundamentales que rige la gramática de una lengua. Por otro lado, el vocabulaio productivo se refiere a la capacidad de un sujeto de producir enunciados que se adecuen a las normas de una lengua (en este ámbito entran la redacción, la capacidad de expresión verbal, la argumentación, entre otras). Si bien es cierto que en todo proceso comunicativo se requieren ambas, diversos test (Casso, 2010) ponen de manifiesto que el vocabulario productivo susbsume al recepctivo, es decir, que es más fácil para un hablante o incluso un aprendiz leer un texto que producirlo.

Estrictamente, el conocimiento léxico en términos de vocabulario productivo como competencia general (Casso, 2010) puede ser comprendido en términos de sinonimia a partir de investigaciones sobre la distancia semántica (Zapico y Vivas, 2014; Zapico y Vivas, 2015 y Zapico, 2015) donde se observó que a mayor indiferenciación de los significados (mayor grado de sinonimia) hay una mayor capacidad léxico-productiva en el sujeto, puesto que es capaz de dar una mayor cantidad de descriptores para conceptos similares, lo que pone de relieve las diferencias de significado.

De la unión de los dos grandes conceptos (la predicción del desmepeño y la competencia léxica) se postula la hipótesis de que un mayor grado de competencia léxica debería redundar en un mayor desempeño, puesto que se estaría en posesión de capacidades de comprensión, análisis, síntesis y comunicación, necesarias para el afrontamiento de 
cualquier texto o trabajo. Asimismo, esta hipótesis ha sido abordada en un estudio previo (Zapico, 2016b), donde se observó una correlación parcial entre ambas variables, aunque dicho estudio puede ser considerado exploratorio por el tamaño de la muestra (40 sujetos) y su sectorización (todos de la carrera de Letras), dado que no puede decirse que se hayan excluido variables, por ejemplo, socio-económicas, que podrían haber tenido incidencia en los resultados.

En estos estudios se han empleado fundamentalmente dos formas de evaluar el desempeño, las que se sostendrán en esta investigación: Una evaluación parcial y continua, que comprende fundamentalmente el análisis de los resultados de las instancias de evaluación parcial, de carácter escrito u oral, calificadas en términos numéricos estándar con la escala 0-10. Esta es la propia de la modalidad de las cursadas en universidades nacionales e institutos de formación docente nacionales Y una evaluación global y puntual que corresponde a lo sucedido con el alumnado al finalizar las distintas cursadas, medidas en términos de éxito-retraso-fracaso (típicamente utilizados para medir el grado de finalización de una carrera donde éxito es terminar la carrera a tiempo de acuerdo con el plan de estudios, retraso es terminar la carrera con al menos un año de atraso respecto al plan de estudios y fracaso es no finalizar la carrera, sin pormenorizar las causas) adaptadas a un modelo dicotómico lineal de éxito-fracaso donde éxito es aprobar la cursada. Estos modelos han sido empleado en multiplicidad de estudios (Hernández, 2005; Marín, Infante y Troyano, 2000; Vargas 2007; Velázquez y Soriano, 2006, entre otros).

Para poner a prueba la hipótesis mencionada anteriormente se llevó a cabo un estudio cuantitativo de carácter correlacional-descriptivo, puesto que se busca observar posibles correlaciones entre dos variables: competencia léxico-productiva (evaluada en términos de sinonimia) y desempeño académico; a partir de la hipótesis parcialmente comprobada de que ambas varían en un sentido positivo (a mayor grado de competencia léxico-productiva mayor desempeño académico) y teniendo en cuenta que esta vez la muestra estuvo compuesta por estudiantes de más de múltiples carreras de ciencias humanas. 


\section{Metodología}

La investigación se dividió en dos partes. En la primera se obtuvo información sobre la competencia léxico-productiva de los sujetos de la muestra en cuestión. En la segunda se observó el rendimiento académico de dichos sujetos a la luz de las puntuaciones obtenidas.

Materiales: Para la elaboración del Z-Test de sinónimos (Zapico, 2016b) se tomaron Las normas de producción de atributos semánticos en castellano rioplatense (Vivas, Comesaña, García Coni, Vivas y Yerro, 2013), a partir de las cuales se obtuvieron 80 palabras de alta frecuencia que poseen sinónimos según WordReference diccionario de sinónimos (2016). Luego, a través de la comparación con la base CREA de las 1000 palabras más frecuentes de la Real Academia Española (2016) y el juicio de pares especialistas en el área, se llegó a una lista final de 40 palabras. La prueba consiste en dar al menos un sinónimo de cada palabra y emplearla en una oración gramaticalmente correcta donde la palabra y su sinónimo sean intercambiables. Por cada acierto se suma un punto, con un mínimo de 0 y un máximo de 40 . Para el análisis de datos se utilizó el software de análisis Statistic Pack for Social Sciences (SPSS).

Muestra: Un total de $\mathrm{N}=120$ estudiantes, un $68,3 \%$ mujeres (82) y un $31,7 \%$ hombres (38), de entre 18 y 30 años de edad con una media de 20,2, de cuatro carreras, que cursaron la materia introductoria de su respectiva disciplina, a saber: Profesorado en Lengua y Literatura, Introducción a la Literatura (30: 34\% hombres, 66\% mujeres); Profesorado en Historia, Introducción a la práctica histórica (30: 50\% hombres, $50 \%$ mujeres); Profesorado de Inglés, Prácticas Discursivas I (30: 24\% hombres, 76\% mujeres); Profesorado en Educación Primaria, Pedagogía (30: 20\% hombres, 80\% mujeres); durante el primer cuatrimestre del año 2016 en el Instituto Nacional de Formación Docente Continua (IFDC).

Procedimiento: Una vez obtenido el consentimiento oral que fue posteriormente respaldado por su versión escrita, se procedió a la administración del Z-Test. Este se llevó a cabo de manera individual en el espacio de trabajo del docente-personal investigador, en condiciones óptimas de iluminación y sonido, sin distractor alguno. El tiempo de administración fue de treinta (30) minutos, aunque ningún sujeto superó dicha medida. 
A partir del conjunto inicial de 120 sujetos y sus puntuaciones en el test de sinónimos, se delimitaron cuatro grupos: $\mathrm{A}(>30$ alta competencia léxica) $\mathrm{B}(20<30$ buena competencia léxica) $\mathrm{C}(10<20$ regular competencia léxica) y $\mathrm{D}(<10$ baja competencia). Una vez definidos los grupos se observó su desmpeño en dos evaluaciones escritas (E1 y E2) y un trabajo escrito integrador (TF).

Análisis de datos: Primero se observó la distribución de los grupos del test en relación con las variables sexo y carrera, analizando alguna posible relación entre dichas variables. Luego se realizó una comparación de la tasa de éxito en la cursada a través de la comparación porcentual del alumnado en un sentido general (la aprobación o no de la cursada) y particular (las tres instancias evaluativas). Además se realizó una comparación de medidas de tendencia central (media y moda) para los resultados obtenidos en: E1, E2 y TF, correlacionadas con los grupos A,B,C y D de desenvolvimiento léxico, junto con las correspondientes medidas de dispersión (coeficiente de variación y mediana). También se aplico el cálculo de la F de Fischer de forma ANOVA (para verificar muestras con una distribución normal, datos independientes, $\mathrm{N}=120$, $\mathrm{k}=4$, g.l $=3$ y un p.valor $<0.05$ ) para determinar el grado de significación estadística de la correlación entre los resultados de las instancias evaluativas y la competencia léxico-productiva.

\section{Resultados y discusión}

A continuación se muestran las distribuciones para los grupos A, B, C y D de competencia léxica, con sus frecuencias absolutas y clasificadas también por género y carrera. 


\section{Tabla 1}

Distribución de los grupos $A, B, C$ y $D$ de conocimiento léxico en función de su frecuencia, la carrera y el sexo

\begin{tabular}{|l|l|l|l|l|l|l|l|l|l|l|l|l|l|}
\hline \multicolumn{10}{|c|}{ Grupos de conocimiento léxico por frecuencia, carrera y sexo } \\
\hline \multicolumn{2}{|c|}{ Grupos Léxico } & \multicolumn{3}{|c|}{ Grupos por Carrera } & \multicolumn{5}{c|}{ Grupos por Sexo } \\
\hline A & 20 & $17 \%$ & & A & B & C & D & & HOMBRE & & MUJER & \\
\hline B & 41 & $34 \%$ & Hist & $16 \%$ & $40 \%$ & $30 \%$ & $14 \%$ & A & 5 & $13 \%$ & 15 & $18 \%$ \\
\hline C & 42 & $35 \%$ & Letr & $20 \%$ & $43 \%$ & $20 \%$ & $16 \%$ & B & 12 & $31 \%$ & 29 & $35 \%$ \\
\hline D & 17 & $14 \%$ & Ingl & $20 \%$ & $23 \%$ & $43 \%$ & $14 \%$ & C & 14 & $37 \%$ & 28 & $34 \%$ \\
\hline Total & 120 & $100 \%$ & Prim & $10 \%$ & $30 \%$ & $43 \%$ & $16 \%$ & D & 7 & $19 \%$ & 10 & $13 \%$ \\
\hline & & & & & & & & Total & 38 & $100 \%$ & 82 & $100 \%$ \\
\hline
\end{tabular}

Nota: Datos de los autores.

Como se observa en la Tabla 1, la distribución general de la competencia léxica se distribuye de acuerdo con estudios previos y con cierta lógica: el grueso de la muestra recae en las categorías B y $\mathrm{C}$, que juntas conforman el $70 \%$ de todos los sujetos. Se condice con la evidencia de que el público usuario del lenguaje, en un nivel superior, goza de una competencia media que es necesaria para el afrontamiento de cualquier estudio. Este mismo patrón se repite tanto en las variables carrera como sexo e indica que no hay diferencias en la distribución a partir de estos factores. En los tres casos los extremos, correspondientes a los grupos A y D (persona usuaria de alta competencia y persona usuaria de baja competencia respectivamente), son ocupados por la minoría de la población que, por motivos que no se han examinado en este trabajo, goza de un nivel de competencia léxico productiva muy grande o muy pequeño.

Hay, además, dos elementos que llaman la atención. En el caso de la distribución por carrera, la realmente baja cantidad de sujetos en el grupo A de Educación Primaria, que está 10\% menos poblado que Letras e Ingles y 6\% debajo de Historia. En el caso de la distribución por sexos la pequeña superioridad que gozan las mujeres en los extremos: tienen un $5 \%$ más de sujetos en el grupo de mejor competencia y un $6 \%$ menos en el grupo de peor competencia. No obstante, las diferencias son poco significativas en términos estadísticos y, al menos con estos datos, no son atribuibles a una causa que no sea el azar. 
En la figura 1 se encuentra expresado el porcentaje de finalización de las cursadas en función de los cuatro grupos definidos, junto con la línea de tendencia.

\section{Figura 1}

Porcentaje de finalización de cursada para los cuatro grupos.

\section{Porcentaje Finalización Cursada}

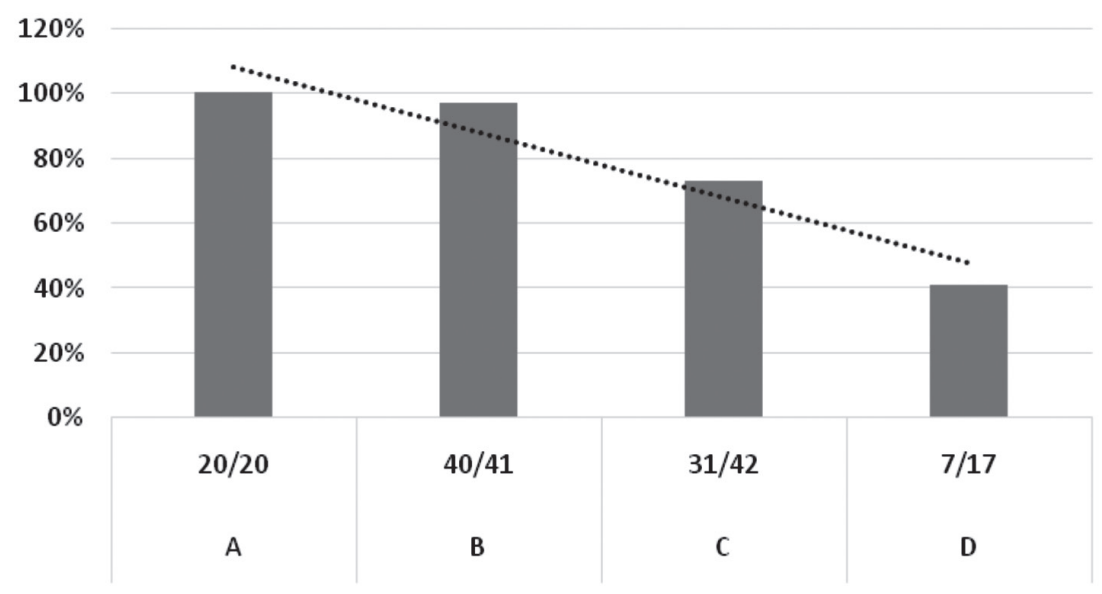

Fuente: Datos de autores.

Ya a partir del análisis de la figura 1 se puede ir delineando algún enunciado respecto a la hipótesis, puesto que la cantidad de sujetos que finalizaron la cursada de manera exitosa decrece de acuerdo con cómo decrece la competencia léxico-productiva. Un $100 \%$ de finalización para el grupo A contrasta con un 40\% de finalización para el grupo D. Esto se ve bien representado, además de por los porcentajes, por la línea de tendencia cuyo mejor ajuste consiste en un modelo $\mathrm{y}=\mathrm{ax}+\mathrm{b}$ o modelo lineal. Aun así, hay que hacer una salvedad importante para el grupo $\mathrm{B}$, que si bien presenta un porcentaje menor de éxito que A ( $100 \%$ vs 97\%) la diferencia real es de apenas un sujeto y podría ser fácilmente atribuible a motivos que no sean el nivel de competencia léxica. No puede decirse lo mismo del grupo $\mathrm{C}$, que presentó un porcentaje de éxito del $73 \%$ que, si bien es alto, es notablemente más bajo que los grupos superiores. 
Esto parece mostrar un agrupamiento del tipo A-B/C-D en cuanto al desempeño global en la cursada. Es decir, el nivel de competencia léxico productiva es importante a la hora de tener un buen desempeño, pero lo determinante es llegar a un "piso" de competencia mínimo que parece ubicarse entre $\mathrm{C}$ y B. Esto se ve reforzado por el dato de que de los once sujetos que no lograron completar sus cursadas, ocho corresponden a puntuaciones más cercanas a 20 (el grupo D) que a 30 (el grupo B). Lo cual nos permitiría situar, hipotéticamente, dicho piso en $\geq 25$. No obstante, esta afirmación debe ser corroborada estadísticamente, lo cual se realizará en futuros estudios.

En la figura 2 se observa la media de los cuatro grupos en cada una de las instancias evaluativas, posteriormente una figura con la media final, la moda, el coeficiente de variación y un box-plot.

\section{Figura 2}

Media de cada uno de los grupos en las tres instancias de evaluación

\section{Media de E1,E2 y TF en cuatro grupos}

10

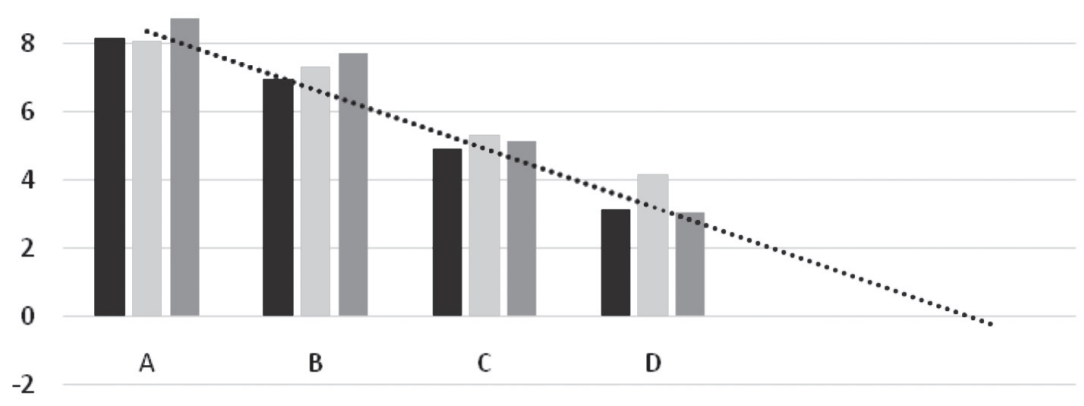

E1
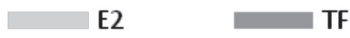

Fuente: Datos de autores.

Consistentemente con la figura 2, la media del desempeño obtenido en las distintas instancias de evaluación también presenta variaciones decrecientes a medida que baja la competencia léxico-productiva. Esta particularidad se mantiene constante para las tres evaluaciones, y, en este caso (a diferencia de lo que sucedía con el desempeño global donde 
prácticamente el grupo A y B eran indiferenciados), la media va descendiendo de manera lineal y constante, lo cual se refleja en la línea de tendencia. A su vez este tipo de distribución era esperable puesto que para lograr la aprobación de la cursada se requieren notas de al menos 4 (cuatro) en las tres instancias, mientras que al analizar las notas obtenidas la diferencia entre A, B, C, y D muestra como al parecer distintos niveles de competencia léxico-productiva inciden sobre el desempeño.

No obstante, es necesario llamar la atención sobre un fenómeno que en parte aparecía en la figura 1, pero que en este caso sucede de otra manera. La diferencia entre B comparada con $\mathrm{C}$ en cuanto a la media de todas las instancias (2,2 puntos) es llamativamente más grande que las diferencias entre A-B (1 punto) y C-D (1,3 puntos). Es decir, encontramos nuevamente un salto cualitativo (no tan marcado, como ya habíamos aclarado) que se sitúa en un punto medio entre $\mathrm{B}$ y $\mathrm{C}$ que explica la gran diferencia que este recorrido presenta (2,2 puntos) en comparación con los otros dos.

\section{Tabla 2}

Estadisticos de media, moda, mediana y coeficiente de variación para el desempeño global de los grupos durante la cursada

\begin{tabular}{|c|c|c|c|c|}
\hline \multicolumn{5}{|c|}{ Estadísticos } \\
\hline Grupo & Media & Moda & Mediana & C. Variación \\
\hline A & 8,3 & 8 & 8,17 & $9,00 \%$ \\
\hline B & 7,3 & 7 & 7,33 & $12,00 \%$ \\
\hline C & 5,1 & 5 & 5,67 & $29,00 \%$ \\
\hline D & 3,3 & 3 & 3,4 & $31,00 \%$ \\
\hline
\end{tabular}

Nota: Datos de autores.

Los datos de la media presentados en la Tabla 2, estadísticamente consistentes a su vez con las figuras anteriores, reflejan un nivel de confianza para la media alto, puesto que las modas de cada uno de los grupos están en valores cercanos a la media, así como la mediana que en cada uno de los casos prácticamente se superpone a la media. Además de que el coeficiente de variación es extremadamente bajo para los grupos A y B, y bajo para los grupos C y D. Incluso el porcentaje de variación que parece subir bastante en los grupos $\mathrm{C}$ y D tiene una explicación sencilla en los datos: en estos dos grupos hay al menos tres 
singularidades (que se visibilizan en el box-plot situado más adelante en el texto) que obtuvieron notas llamativamente altas en comparación a la media. Esto puede ser corroborado teniendo en cuenta que los valores de la mediana son en cada caso cercanos a la media, lo cual señala un nivel de dispersión de los datos mínimo. Y considerando que la media para los grupos $\mathrm{C}$ y D es bastante baja, basta un buen desempeño para que el coeficiente de variación suba considerablemente.

Dicho esto, volvemos a encontrar una baja en la media de las calificaciones obtenidas de acuerdo con el grado de competencia léxico-productivo de los sujetos. Incluso en la Tabla 2, al analizar el coeficiente de variación, vuelve a llamar la atención, sobremanera, el salto cualitativo que se da en el trayecto B-C (17\%) que supera con creces el propio de los trayectos A-B (3\%) y C-D(2\%). Esto se explica porque en el caso de los grupos A y B no hallamos singularidades. Es decir, en los niveles de competencia léxico-productiva alto y bueno no se presentan casos donde los sujetos hayan obtenido notas excesivamente bajas. Esto indicaría nuevamente un punto (un mínimo puntaje en el Z-Test) situado entre el grupo B (donde todos los sujetos tienen rendimiento de acuerdo con la media, sin irregularidades) y C (donde empiezan a encontrarse casos de desempeños irregulares, en el grupo hay puntuaciones fluctuantes) que parece garantizar un rendimiento estable. 


\section{Figura 3}

Box-plot con la presentación de singularidades

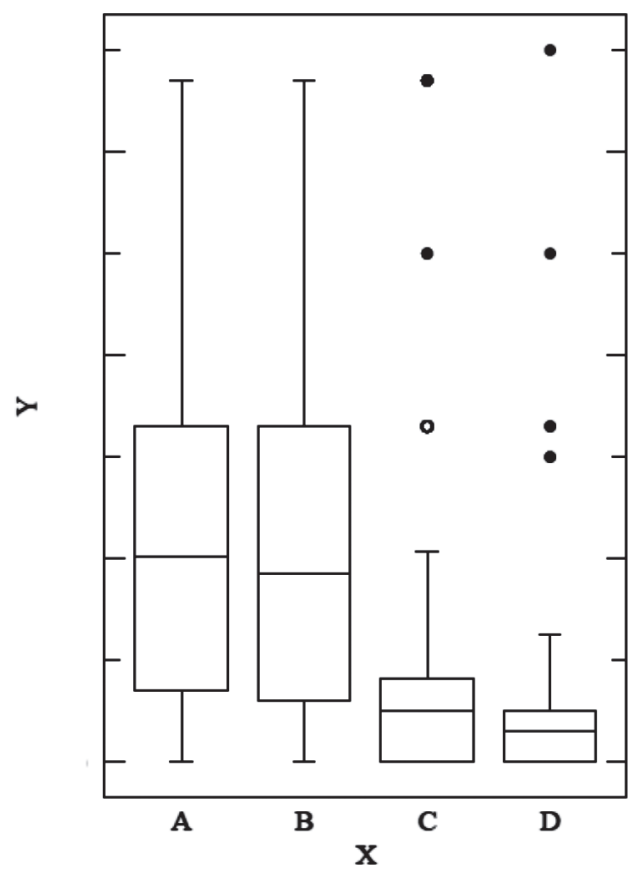

Fuente: Datos de autores.

Finalmente se muestran los resultados de la prueba ANOVA para el análisis de correlación entre desempeño académico y competencia léxico-productiva., en la Tabla 3

\section{Tabla 3}

Resultados de la prueba ANOVA para correlación entre grupo léxico y desempeño general en la cursada

\begin{tabular}{|l|c|c|c|c|c|c|}
\hline \multicolumn{1}{|c|}{ Resultado de prueba ANOVA } \\
\hline F.Var & S.C & G.L & S.C & F & p.valor & p. critico \\
\hline Entre & 3821. & 3 & 1274. & 2.639 & $0.04^{*}$ & 105 \\
\hline Error & $1,13 \mathrm{E}+09$ & 234 & 482.7 & \multicolumn{5}{|c|}{237} \\
\hline Total & $1,17 \mathrm{E}+09$ & \multicolumn{7}{|c|}{} \\
\hline
\end{tabular}

*The probability of this result,assuming the null hypothesis, is 0.04

Nota: Datos de autores. 
En la Tabla 3 se observa que las diferencias de media observadas anteriormente adquieren significancia estadística, puesto que el estadístico devuelve un p.valor de 0.04 lo cual se sitúa dentro del área de rechazo de Ho (0.05) y nos permite afirmar que, al menos en esta muestra, hay una correlación entre la competencia léxico-productiva y el desempeño académico. Este resultado era en parte esperable puesto que las diferencias fueron notorias tanto en los planos más generales (la tasa de éxito y la media de desempeño) como particulares (las instancias particulares de evaluación), y en todos los casos se observó una tendencia que situaba los altos desempeño con las altas puntuaciones en el Z-Test, mientras que las puntuaciones bajas (salvo contadas singularidades) conllevaron desempeños también bajos.

\section{Conclusiones}

Hemos observado las relaciones entre la competencia léxico-productiva y el desempeño académico, lo cual ha puesto de relieve algunos datos interesantes que es apropiado destacar.

Sobre la distribución se ha hallado que esta es uniforme y se ajusta a un diagrama de campana, no se encontraron diferencias significativas a partir de la carrera o el sexo. Aun así hay una pequeña diferencia entre hombres y mujeres en los extremos que se estudiará en futuras investigaciones.

Sobre la tasa de éxito hay que decir que es evidente la relación que existe entre altos niveles de competencia léxico-productiva y desempeño, lo que llega al punto en el cual se pudo delimitar (de manera hipotética y provisoria) un punto crítico entre las puntuaciones $20 \mathrm{y}$ 30 donde hay un salto cualitativo en el desempeño. Este punto crítico también fue observado en la diferencia entre medias y los coeficientes de correlación, y manifiesta una importancia cabal en la explicación de distintos desempeños (dado que la gran varianza para grupos de media o baja competencia léxica no nos otorga certidumbre respecto a un posible desempeño, mientras que los grupos de buena y alta competencia tienen una varianza baja y el rendimiento es más estable, lo cual confiere mayor grado de predictibilidad). Esto abre la puerta para futuras investigaciones sobre este intervalo, que podría resultar valioso a efectos de establecer objetivos mínimos para la evaluación del léxico en cursos de ingreso o materias de primeros años en carreras de nivel superior. 
Sobre la media general y la correlación positiva encontrada a través de la significancia estadística, parece confirmar la hipótesis de que a mayor grado de competencia léxico-productiva hay un mayor desempeño académico. En este estudio se ha podido observar que esto es válido independientemente de una serie de carreras de ciencias humanas, e independientemente del sexo de los sujetos (recordemos que la particularidad hallada hablaría sobre la competencia previa mayor que trae el sexo femenino, no sobre la incidencia del sexo en la correlación entre variables).

No obstante, aún falta ahondar en otras variables que podrían ser importantes tales como: la condición del alumnado en relación con el trabajo (si trabaja a tiempo completo, parcial, o no trabaja), el nivel socio-económico (la facilidad de acceso a materiales y bienestar podría incidir en el desempeño), la motivación (autoestima, expectativas respecto al futuro, etc.), entre otras. A su vez, también hay que poner a prueba la hipótesis en carreras de ciencias exactas, donde el uso del lenguaje no es tan directo, para poder delimitar en qué contextos académicos es válida la correlación.

Por último, aunque no menos importante, recordar que hablar de competencia léxico-productiva no es muy diferente de hablar de capacidad de comunicación y expresión. La posibilidad de poner palabras a nuestros sentimientos, deseos, necesidades, dolores es algo que trasciende el espacio académico y se relaciona también con nuestra posibilidad de entablar mejores relaciones, tanto con el propio sujeto mismo como con los otros.

\section{Referencias}

Álvarez Mendez, J. (2001). Evaluar para conocer, examinar para excluir. Madrid: Morata.

Arribas, J. (2012) El rendimiento académico en función del sistema de evaluación empleado. Revista RELIEVE, 18(1), 2-11.

Barsky, O, Sigal, V, y Dávila, M. (2012). Los desafios de la universidad argentina. Buenos Aires: Siglo XXI.

Benitez, M, Gimenez, M, y Osicka, R. (2000) Las asignaturas pendientes y el rendimiento académico.¿Existe alguna relación? Revista Educación, 189(1), 224-235. 
Buscà, F., Cladellas, L., Calvo, J., Martín, M., Padrós, M. y Capllonch, M. (2011) Evaluación formativa y participativa en docencia universitaria. Un estudio sobre los artículos publicados en revistas españolas entre 1999 y 2009. Aula Abierta, 39(2), 137-148.

Casso, J. (2010). Análisis y revisión críticas de los materiales de evaluación de la competencia léxica. Elaboración de un test de vocabulario de nivel umbral. Nebrija: Editorial del Departamento de Lenguas Aplicadas.

Cozza, E. (1998) Calidad académica y evaluación universitaria: Una nueva sinonimia. Revista Cientifica UBP, 4(11), 11-19.

Garaigordobil, M y Torres, E. (1996) Evaluación de la creatividad en sus correlatos con inteligencia y rendimiento académica. Revista de Psicología Universitas Tarraconesis, 18(1), 87-98.

García de Fanelli, A. (2014) Rendimiento académico y abandono universitario. Revista Argentina de Educación Superior, 1(8), 9-38.

Giovagnoli, P. (2001) Determinantes de deserción y graduación universitaria (Tesis de licenciatura). Universidad de La Plata, Ciudad de La Plata, Argentina. Recuperada de http://sedici.unlp.edu.ar/ handle/10915/37129

González-Pérez, M. (2000) La evaluación del aprendizaje: Tendencias y reflexión crítica. Revista Cubana de Educación Superior, 20(1), 47-67.

Hernández, A. (2005) La motivación en estudiantes universitarios. Actualidades Investigativas en Educación, 5(2), 123-135.

Jiménez, V., Izquierdo, J. y Blanco, A. (2000) La predicción del rendimiento académico: Regresión lineal versus regresión logística. Revista Psicothema, 12(2), 248-252.

López, V. (2008). Desarrollando sistemas de evaluación formativa y compartida en la docencia universitaria. Análisis de resultados de su puesta en práctica en la formación inicial del profesorado. European Journal of Teacher Education, 31(3), 293-311.

Lugo, E, Villatoro, J, Medina-Mora, E y García, F. (1996) Autopercepción del rendimiento académico en estudiantes méxicanos. $R e$ vista Méxicana de Psicología, 13(1), 37-47.

Marín, M., Infante, E. y Troyano, Y. (2000). El fracaso académico en la universidad: Aspectos motivacionales e intereses profesionales. Revista Latinoamericana de Psicología, 32(3), 505-517. 
Meerbeke, A. y González, C. (2005) Factores asociados al rendimiento académico en estudiantes de medicina. Revista Educación Médica, 8(2), 74-82.

Navarro, R. (2003) El rendimiento académico: Concepto, investigación y desarrollo. REICE, 1(2), 1-13.

Pérez, E. (2002) Validación de características al ingreso como predictores del rendimiento académico en la carrera de medicina. Revista Cubana de Educación Superior, 16(1), 5-18.

Pérez, M. (2001) La evaluación del aprendizaje: Tendencias y reflexión crítica. Revista Cubana de Eduación Superior, 15(1), 85-96.

Pozo, M. y Álvarez, O. (2007) Desempeño académico de universitarios en relación con ansiedad escolar y auto-evaluación. Revista Acta Colombiana de Psicología, 11(1), 13-23.

RAE. (2016). Diccionario. Disponible en http://dle.rae.es

Renault, G, Kohan, N y Solano, A. (2001) Factores que intervienen en el rendimiento académico de los estudiantes de psicología y psicopedagogía. Revista Signos Universitarios, 1(1), 27-34.

Tejedor, F. y Repiso, A. (2007) Causas del bajo rendimiento del estudiante universitario (en opinión de los profesores y alumnos). Propuestas de mejora en el marco del EEES. Revista de Educación, 1(342), 443-473.

Vargas, G. (2007) Factores asociados al rendimiento académico en estudiantes universitarios, una reflexión desde la calidad de la educación superior pública. Revista Educación, 31(1), 43-63.

Velázquez, L. y Soriano, N. (2006) Rendimiento académico y contexto familiar en estudiantes universitarios. Enseñanza e Investigación en Psicología, 11(2), 255-270.

Vélez, E., Schiefelbein, E. y Valenzuela, J. (1994) Factores que afectan el rendimiento académico en la educación primaria. Revista latinoamericana de innovaciones educativas en Argentina, 1(17), $35-48$.

Vivas, J., Comesaña, A., García Coni, A., Vivas, L. y Yerro, M. (2013). Psicología y otras ciencias del comportamiento. Compendio de investigaciones actuales I. Libertador San Martin: Editorial Universidad Adventista del Plata.

Word Reference. (2014.) Diccionario de sinónimos. Recuperado de www.wordreference.com/es 
Zapico, G. y Vivas, J. (2014). La sinonimia como caso particular de distancia semántica. Encontros Bibli, 20(40), 253-266.

Zapico, G. y Vivas, J. (2015) La sinonimmia desde una perspectiva lingüistico-cognitiva. Medición de la distancia semántica. Onomázein, 32, 198-211. doi:10.7764/onomázein.32.11

Zapico, M. (2016a). Evaluación de estrategias de enseñanza orientadas a competencias: Una comparación de caso entre estilo orientado a la competencia y el orientado al contenido. Revista Ensayos Pedagógicos, 11(1), 135-146.

Zapico, M. (2016b). Evaluación de desempeño académico: La competencia léxica productiva como competencia con validez predictiva. Revista Ensayos Pedagógicos, 11(2), 159-171.

Zapico, M. (2015). Análisis de las distancias semánticas entre conceptos. La sinonimia. Revista Psiciencia, 1, 282-285. doi: 10.5872/ psiencia/7.1.041011 